\title{
Random walk in a stratified independent random environment
}

\author{
Brémont Julien*
}

\begin{abstract}
We study Markov chains on a lattice in a codimension-one stratified independent random environment, exploiting results previously established in [2]. The random walk is first shown to be transient in dimension at least three. Focusing on dimension two, we provide sharp sufficient conditions for either recurrence or transience. We determine the critical scale of the local drift along the strata, corresponding to the frontier between the two regimes.
\end{abstract}

Keywords: Markov chain; recurrence; stratification; random environment; independence. AMS MSC 2010: 60G17; 60J10; 60K37.

Submitted to ECP on November 14, 2018, final version accepted on June 21, 2019.

\section{Introduction}

A very first and important question concerning the asymptotic behaviour of a Markov chain on a lattice in inhomogeneous environment is the question of the recurrence/transience. We consider in the present paper the situation where the environment is stratified, continuing the line of research initiated by Matheron and de Marsily [13] in the early 1980s. Such a model of random motion was initially motivated by hydrology and the diffusion of pollutants in the ground. The latter is porous and stratified for geologic reasons, with some heterogeneity among the strata. In 2003, a discrete Markov chain version was introduced by Campanino and Petritis [3], with a different inspiration, coming from physical considerations about the study of discrete gauge theories.

Let us detail the model studied in [2], which is an extension of the one originally introduced in [3]. Fixing $d \geq 1$, we consider a Markov chain $\left(S_{n}\right)_{n \geq 0}$ in $\mathbb{Z}^{d} \times \mathbb{Z}$, with $S_{0}=0$. In the sequel, quantities relative to the first (resp. second) coordinate in $\mathbb{Z}^{d}$ (resp. $\mathbb{Z}$ ) are declared "horizontal" (resp. "vertical"). We assume that the transition laws only depend on the vertical coordinate, i.e. are constant on each affine hyperplane (or stratum) $\mathbb{Z}^{d} \times\{n\}, n \in \mathbb{Z}$.

Let $B_{d}(a, r)$ be the Euclidean ball in $\mathbb{R}^{d}$ of center $a$ and radius $r$. A vector $x \in \mathbb{Z}^{d}$ is written in column, with transpose $x^{t}$. For each vertical $n \in \mathbb{Z}$, suppose to be given positive reals $p_{n}, q_{n}, r_{n}$, with $p_{n}+q_{n}+r_{n}=1$, and a probability measure $\mu_{n}$ with support in $\mathbb{Z}^{d}$, satisfying:

\footnotetext{
${ }^{*}$ Université Paris-Est Créteil, France. E-mail: julien . bremont@u-pec. fr
} 
Hypothesis 1.1. Let $d \geq 1$. There exists $\eta>0$ such that for all vertical $n \in \mathbb{Z}$ :

$-\min \left\{p_{n}, q_{n}, r_{n}\right\} \geq \eta$,

$-\operatorname{Supp}\left(\mu_{n}\right) \subset B_{d}(0,1 / \eta) \cap \mathbb{Z}^{d}$,

- the spectrum of the real non-negative symmetric matrix $\sum_{k \in \mathbb{Z}^{d}} k k^{t} \mu_{n}(k)$ is included in $[\eta,+\infty)$. When $d=1$, this condition is replaced by $\mu_{n}(0) \leq 1-\eta$.

In [2], we assumed no link between the data corresponding to different vertical $n$. The second condition on the support of $\mu_{n}$ was replaced by a weaker one (a uniform in $n$ moment condition). For the sake of simplicity, we restrict here to the above setting. The transition laws of $\left(S_{p}\right)_{p \geq 0}$ are now defined, for all $(m, n) \in \mathbb{Z}^{d} \times \mathbb{Z}$ and $k \in \mathbb{Z}^{d}$, by:

$$
(m, n) \stackrel{p_{n}}{\longrightarrow}(m, n+1),(m, n) \stackrel{q_{n}}{\longrightarrow}(m, n-1),(m, n) \stackrel{r_{n} \mu_{n}(k)}{\longrightarrow}(m+k, n) .
$$

With a picture:

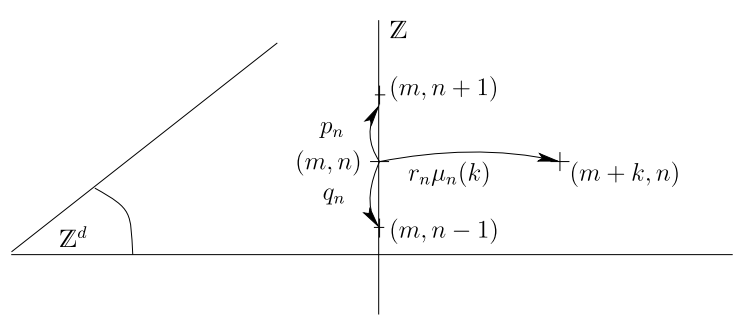

The set of transition laws is also called the "environment". An important submodel, called "vertically flat" in the sequel corresponds to the symmetry assumption $p_{n}=q_{n}$, $n \in \mathbb{Z}$

For the general model, an important observation (appearing in [3]), following from the invariance of the environment under horizontal translations, is that the vertical component of $\left(S_{n}\right)$, restricted to vertical jumps, forms a Markov chain on $\mathbb{Z}$. The latter is called the "vertical random walk" in this article. Its transition laws are given by:

$$
n \stackrel{p_{n} /\left(p_{n}+q_{n}\right)}{\longrightarrow} n+1, n \stackrel{q_{n} /\left(p_{n}+q_{n}\right)}{\longrightarrow} n-1 .
$$

The Markovian character of the vertical component is not true in general for an inhomogeneous Markov chain, when the environment is not stratified. This gives to $\left(S_{n}\right)$ some kind of "product structure", essential in the analysis. For example, for $\left(S_{n}\right)$ to be recurrent, it is necessary that the vertical random walk is recurrent, the conditions for which being classical. Assuming these, in order to study the recurrence of $\left(S_{n}\right)$ one roughly has to focus on the horizontal displacement.

The "local horizontal drift" in the stratum $n \in \mathbb{Z}$, the expectation of $\mu_{n}$, is $\varepsilon_{n}:=$ $\sum_{k \in \mathbb{Z}^{d}} k \mu_{n}(k)$. Let us now present former results concerning recurrence/transience for this model.

Consider first the vertically flat case. In this situation, the vertical random walk is the (recurrent) simple random walk on $\mathbb{Z}$. The Campanino-Petritis model [3] consists in taking $d=1$, with $p_{n}=q_{n}=r_{n}=1 / 3$ and $\mu_{n}=\delta_{\alpha_{n}}$, fixing some sequence $\left(\alpha_{n}\right)_{n \in \mathbb{Z}} \in\{ \pm 1\}^{\mathbb{Z}}$ and where $\delta_{x}$ is Dirac measure at $x$. It is shown in [3] that $\left(S_{n}\right)$ is recurrent when $\alpha_{n}=(-1)^{n}$ and transient when $\alpha_{n}=1_{n \geq 1}-1_{n \leq 0}$ or when $\left(\alpha_{n}\right)$ are typical realizations of independent and identically distributed (i.i.d for the sequel) random variables with $\mathbb{P}\left(\alpha_{n}= \pm 1\right)=1 / 2$. Many works followed, close to this setting. Guillotin-Plantard and Le Ny [9] showed transience results when the $\left(\alpha_{n}\right)$ are independent with marginals described by some dynamical system. Pene [15] proved transience when the $\left(\alpha_{n}\right)$ are stationary, under a decorrelation condition. Castell, Guillotin-Plantard, Pene and Schapira [5] quantified transience when the $\left(\alpha_{n}\right)$ are i.i.d., studying the tail of the 
annealed return time to 0 . Still under the condition $p_{n}=q_{n}, n \in \mathbb{Z}$, Devulder and Pene [7] considered an extension of the model in [3] and established transience when the $\left(r_{n}\right)$ are i.i.d. non-constant and $\mu_{n}=\delta_{\alpha_{n}}$, with arbitrary $\left(\alpha_{n}\right)$. In [4], Campanino and Petritis, focusing on the initial model of [3], studied a random perturbation of a periodic $\left(\alpha_{n}\right)$.

In [1], Theorem 1.2, for the general vertically flat case with $d=1$, a complete recurrence criterion was given. In this situation, the asymptotics of the random walk is governed by a cocycle related to the local horizontal drift, namely $\left(r_{0} \varepsilon_{0} / p_{0}+\cdots+\right.$ $\left.r_{n-1} \varepsilon_{n-1} / p_{n-1}\right)$ when $n \geq 1$, to be understood as a horizontal flow associated with the environment. More precisely comes into play a two-variables function $\Phi(a, b)$ (see Definition 2.4 below, in the general case), for vertical $a<b$, measuring the "horizontal dispersion" of the previous horizontal flow between vertical levels $a$ and $b$. The quantity deciding for the recurrence/transience of $\left(S_{n}\right)$ quantifies some "capacity of dispersion to infinity" of the environment. It is interesting to notice that it involves the level lines of the function $\Phi(a, b)$ and some notion of curvature at infinity of these lines. A geometric point of view of the recurrence criterion has to be developed. Notice that the form of the criterion in [1], a priori a little abstract, in fact directly comes from the computation of a Poisson kernel in a half-plane. Such a kernel appears in the recurrence criterion for i.i.d. random walks on $\mathbb{Z}^{d}$ (see Spitzer [19] or Ornstein [14]), the latter being an essential ingredient in the proof in [1].

Still for the general vertically flat model when $d=1$, after the principal result of [1], some (little) extra work is required to treat concrete examples. This was done at the end of [1], giving rather fine recurrent quasi-periodic examples ([1], Prop. 1.5; cf also [2], Prop. 7.1). It is also shown that in the vertically flat case, simple random walk in the plane is in some sense the most recurrent one. As is well known, it is hardly recurrent. For instance, roughly a growth condition like $(\log n)^{1+\delta}$ on $\left(r_{0} \varepsilon_{0} / p_{0}+\cdots+r_{n-1} \varepsilon_{n-1} / p_{n-1}\right)$ is sufficient for transience. This explains to some extent the prevalence of transience results in the litterature.

In order to try to enlarge the recurrence domain, it is natural to focus on the general model introduced above. A recurrence criterion was shown in [2], Theorem 2.4, with exactly the same form as in [1], coming from a Poisson kernel. The function $\Phi(a, b)$ (cf Definition 2.4 below) is just a little more complicated. The criterion highlights that the environment defines a new metrization of $\mathbb{Z}^{d} \times \mathbb{Z}$. Let us point out that contrary to the vertically flat case, the vertical random walk can be an arbitrary nearest-neighbour random walk on $\mathbb{Z}$. For instance it can be positive recurrent. In this case, $\left(S_{n}\right)$ is strongly pushed towards $\mathbb{Z}^{d} \times\{0\}$ and in fact nearly behaves as if it were in $\mathbb{Z}^{d}$; see section 7.3 on the "half-pipe" in [2]. Several examples of recurrent and transient random walks were given in [2], in dimensions both 2 and 3 ( $d=1$ or 2 ). In dimension $\geq 4$ (i.e. $d \geq 3$ ), the random walk is always transient ([2], Prop. 2.5, 1)i)).

The purpose of the present article is to extend the applications of [2], studying in detail the previous general model in the important case when the transition laws are random and independent, with a quenched point of view, i.e. for almost-every realization of the environment. On such a model, let us mention a result by Kochler in his doctoral thesis [12], concerning the case when $\mathbb{P}\left(\mu_{n}=\delta_{1}\right)=\mathbb{P}\left(\mu_{n}=\delta_{-1}\right)=1 / 2$, hence giving $\mathbb{P}\left(\varepsilon_{n}= \pm 1\right)=1 / 2$, with $r_{n} /\left(p_{n}+q_{n}\right)=c$ (a constant independent on $n$ ) and $\left(p_{n} / q_{n}, \mu_{m}\right)_{(n, m) \in \mathbb{Z}^{2}}$ independent and identically distributed. Transience is established, after a long (80 p.) and delicate analysis of the Brownian path. Our goal is to recover such a result and try to touch the frontier between recurrence and transience for the present model. This gives an indication on what should happen for more general models of random walks in independent environments. 


\section{Independent setting: presentation and results}

We now restrict to the independent setting. For the rest of the article, we assume that $\left(p_{n}, q_{n}, r_{n}\right)_{n \in \mathbb{Z}}$ are random variables such that $\left(p_{n} / q_{n}\right)_{n \in \mathbb{Z}}$ are i.i.d., with arbitrary or random $\mu_{n}$, verifying Hypothesis 1.1, a.-s.. We first recall the situation, including the vertically flat case, and next present our results. In the sequel, randomness is always for the environment. We never enter the mechanism of the random walk itself (this was done in $[1,2])$.

Proposition 2.1. If either $d \geq 2$ or $\mathbb{E}\left(\log \left(p_{0} / q_{0}\right)\right) \neq 0$, then for a.-e. realization, $\left(S_{n}\right)$ is transient.

It is classical that the condition $\mathbb{E}\left(\log \left(p_{0} / q_{0}\right)\right) \neq 0$ implies the transience of the vertical random walk (see Solomon [18]), from which that of $\left(S_{n}\right)$ follows. For $d \geq 3$, as said above, this is a general result ([2], Prop. 2.5, 1) $i)$ ). The remaining case, i.e. $d=2$ with $\mathbb{E}\left(\log \left(p_{0} / q_{0}\right)\right)=0$, which includes the case when $p_{0}=q_{0}$, a.-s., will be proved in section 3.2. Recall here in passing the related conjecture that any random walk in i.i.d. random environment in $\mathbb{Z}^{3}$ is transient, supposing ellipticity conditions on the data; see Kalikow [11] and Sabot [16].

Suppose next that $d=1$ and $\mathbb{E}\left(\log \left(p_{0} / q_{0}\right)\right)=0$. When $p_{0} / q_{0}=1$, a.-s., (notice then that $\left.r_{n}=1-2 p_{n}\right)$ i.e. for the vertically flat case, by [1] Prop. $\left.1.4 i\right)$, then $\left(S_{n}\right)$ is recurrent whenever:

$$
\left|\sum_{-n \leq k \leq 0} r_{k} \varepsilon_{k} / p_{k}\right|+\left|\sum_{0 \leq k \leq n} r_{k} \varepsilon_{k} / p_{k}\right|=O\left((\log n)^{1 / 2}\right) .
$$

Still when $p_{0} / q_{0}=1$, a.-s., in the other direction, when applying [1] Prop. 1.6 we obtain that if the $\left(r_{n} \varepsilon_{n} / p_{n}\right)_{n}$ are $i . i . d$ non-constant random variables, then, for a.e. realization, $\left(S_{n}\right)$ is transient. This slightly extends in the elliptic setting former results of [3] and [7]. When the $\left(r_{n} \varepsilon_{n} / p_{n}\right)_{n}$ are only independent, the critical growth for $\sum_{0 \leq k<n} r_{k} \varepsilon_{k} / p_{k}$ with respect to recurrence/transience is expected to be $\log n$, thus somehow corresponding to $\varepsilon_{n}$ of order $1 / n$.

We turn next to the case when $d=1$ and $\mathbb{P}\left(p_{0} / q_{0}=1\right)<1$. The vertical random walk is now Sinaï's random walk [17], very different from simple random walk and with a typical scale of order $(\log n)^{2}$ at time $n$. We need to introduce some notations, the first ones concerning the exponential of the classical potential governing the behaviour of Sinaï's random walk.

Definition 2.2. Set $a_{n}=q_{n} / p_{n}, n \in \mathbb{Z}$, and:

$$
\rho_{n}=\left\{\begin{array}{cc}
a_{1} \cdots a_{n}, & n \geq 1 \\
1, & n=0 \\
\left(a_{n+1} \cdots a_{-1} a_{0}\right)^{-1} & n \leq-1 .
\end{array}\right.
$$

For $n \geq 0$, introduce the quantities:

$$
v_{+}(n)=\sum_{0 \leq k \leq n} \rho_{k} \text { and } v_{-}(n)=a_{0} \sum_{-n-1 \leq k \leq-1} \rho_{k} .
$$

In the same way, let for $n \geq 0$ :

$$
w_{+}(n)=\sum_{0 \leq k \leq n} 1 / \rho_{k} \text { and } w_{-}(n)=\left(1 / a_{0}\right) \sum_{-n-1 \leq k \leq-1} 1 / \rho_{k} .
$$

Notice for the sequel that $\rho_{n+1} / \rho_{n}=a_{n+1} \in[\eta, 1 / \eta], n \in \mathbb{Z}$. If $\mathbb{E}\left(\log \left(p_{0} / q_{0}\right)\right)=0$, then almost-surely, $v_{+}(n)$ and $v_{-}(n)$ increasingly tend to $+\infty$, as $n \rightarrow+\infty$. Indeed, $\log \rho_{n}$ is an i.i.d. random walk with integrable and centered step, hence is recurrent, so $\rho_{n}$ does not go to 0 . 
We also require notations for functions having comparable orders and for inverse functions. Let us first set $\mathbb{N}=\{0,1, \cdots\}$.

Definition 2.3. i) Let $f, g: \mathbb{N} \rightarrow \mathbb{R}_{+}$. We write $f \asymp g$ or $f(n) \asymp g(n)$ if there exists $C>0$ so that for large $n,(1 / C) f(n) \leq g(n) \leq C f(n)$. Set $g \preceq f$ or $g(n) \preceq f(n)$ if $g(n) \leq C f(n)$ for large $n$.

ii) Let $f: \mathbb{N} \rightarrow \mathbb{R}_{+}$be increasing with $\lim f(n)=+\infty$. For large $x \in \mathbb{R}_{+}$, let:

$$
f^{-1}(x)=\max \{n \in \mathbb{N} \mid f(n) \leq x\},
$$

convening that $\max \emptyset=0$. Notice that $f\left(f^{-1}(x)\right) \leq x<f\left(f^{-1}(x)+1\right)$, for large $x \in \mathbb{R}_{+}$. Also $f^{-1}(f(n))=n$, for large $n \in \mathbb{N}$.

As in [2], Definition 2.3, the average horizontal macrodispersion of the environment is described by functions $\Phi_{s t r}(n), \Phi(-m, n), \Phi(n)$ and $\Phi_{+}(n)$. We will only need the last three functions when $d=1$ and these respectively correspond to $\Phi_{u}(-m, n), \Phi_{u}(n)$ and $\Phi_{u,+}(n)$ in [2], Definition 2.3, with $u=1 \in \mathbb{R}_{+}$and $\varepsilon_{s}=m_{s}$, with the notations of [2].

Definition 2.4. i) The structure function, depending only on the vertical, is defined for $n \geq 0$ by:

$$
\Phi_{s t r}(n)=\left(n \sum_{-v_{-}^{-1}(n) \leq k \leq v_{+}^{-1}(n)} \frac{1}{\rho_{k}}\right)^{1 / 2} .
$$

2) For $d=1$ and $m, n \geq 0$, introduce:

$$
\Phi(-m, n)=\left(\sum_{-v_{-}^{-1}(m) \leq k \leq \ell \leq v_{+}^{-1}(n)} \rho_{k} \rho_{\ell}\left[\frac{1}{\rho_{k}^{2}}+\frac{1}{\rho_{\ell}^{2}}+\left(\sum_{s=k}^{\ell} \frac{r_{s} \varepsilon_{s}}{p_{s} \rho_{s}}\right)^{2}\right]\right)^{1 / 2} .
$$

For $n \geq 0$, set:

$$
\Phi(n)=\Phi(-n, n) \text { and } \Phi_{+}(n)=\sqrt{\Phi^{2}(-n, 0)+\Phi^{2}(0, n)} .
$$

3) For $d=1$ and $n \geq 0$, set:

$$
C(n)=\sum_{-v_{-}^{-1}(n) \leq k \leq v_{+}^{-1}(n)} \frac{\varepsilon_{k}}{\rho_{k}} .
$$

We are now in position for stating the main result of this paper, completing the study in the remaining situation when $d=1, \mathbb{E}\left(\log \left(p_{0} / q_{0}\right)\right)=0$ and $\mathbb{P}\left(p_{0} / q_{0}=1\right)<1$.

Theorem 2.5. Let $d=1$, with random variables $\left(p_{n}, q_{n}, r_{n}\right)_{n \in \mathbb{Z}}$ and deterministic or random probability measures $\mu_{n}$, such that for some $\eta>0$ and all $n \in \mathbb{Z}, \min \left\{p_{n}, q_{n}, r_{n}\right\} \geq$ $\eta, \operatorname{supp}\left(\mu_{n}\right) \subset[-1 / \eta, 1 / \eta] \cap \mathbb{Z}$ and $\mu_{n}(0) \leq 1-\eta$. Set $\varepsilon_{n}=\sum_{k \in \mathbb{Z}} k \mu_{n}(k)$. We also assume that the $\left(p_{n} / q_{n}\right)_{n \in \mathbb{Z}}$ are $i . i . d$. with $\mathbb{E}\left(\log \left(p_{0} / q_{0}\right)\right)=0$ and $\mathbb{P}\left(p_{0} / q_{0}=1\right)<1$.

i) For a.-e. realization, $\left(S_{n}\right)$ is recurrent, whenever $\left(\mu_{n}\right)_{n \in \mathbb{Z}}$ is a deterministic sequence verifying:

$$
\sum_{n \in \mathbb{Z}} \frac{\left|\varepsilon_{n}\right|}{\rho_{n}}<+\infty
$$

This condition holds if for some $\delta>0, \varepsilon_{n}=O\left(\exp \left(-|n|^{1 / 2+\delta}\right)\right)$, as $|n| \rightarrow+\infty$.

ii) For a.-e. realization, $\left(S_{n}\right)$ is transient, whenever $\left(\mu_{n}\right)_{n \in \mathbb{Z}}$ is a deterministic sequence verifying $\varepsilon_{n} \geq 0$ for large $n \in \mathbb{Z}$, with:

$$
\sum_{n \in \mathbb{Z}} \frac{\varepsilon_{n}}{\rho_{n}}=+\infty \text { and } \sum_{n \geq N_{0}} \frac{1}{n C(n)}<+\infty,
$$


where $N_{0} \geq 0$ is such that $C(n)>0$, for $n \geq N_{0}$. These conditions hold if for some $\delta>0$ and for large enough $n \in \mathbb{Z}, \varepsilon_{n} \geq \exp \left(-|n|^{1 / 2-\delta}\right)$.

iii) Let the $\left(\varepsilon_{n}\right)_{n \in \mathbb{Z}}$ be independent random variables, independent from the $\left(p_{n}, q_{n}, r_{n}\right)_{n \in \mathbb{Z}}$, such that there exists $1 / 2>\delta>0$, so that for large $n \in \mathbb{N}$, the support of $\varepsilon_{n}$ is a finite set $\left\{\alpha_{i, n}\right\}_{1 \leq i \leq L_{n}} \subset[-1 / \eta, 1 / \eta]$, with $L_{n} \geq 2$, verifying for all $1 \leq i \neq j \leq L_{n}$ :

$$
\left|\alpha_{i, n}-\alpha_{j, n}\right| \geq \exp \left(-n^{1 / 2-\delta}\right) \text { and } \mathbb{P}\left(\varepsilon_{n} \neq \alpha_{i, n}\right) \geq n^{-\delta / 5} .
$$

Then for a.-e. realization, $\left(S_{n}\right)$ is transient.

Remark. In $i)$, the condition $\sum_{n \in \mathbb{Z}}\left|\varepsilon_{n}\right| / \rho_{n}<+\infty$ for recurrence can be interpreted as a condition of "finite dispersion to infinity". It is of different nature than $\sum_{n \in \mathbb{Z}} 1 / \rho_{n}<+\infty$, of "finite channel capacity", defining the half-pipe of [2], section 7.3. This last condition, not true here (as $\rho_{n}$ does not go to 0 , as $|n| \rightarrow+\infty$ ), implied that $\left(S_{n}\right)$ is transient if and only if $\sum_{s \in \mathbb{Z}} r_{s} \varepsilon_{s} /\left(p_{s} \rho_{s}\right) \neq 0$; $\operatorname{cf}[2]$, Prop. 7.4, when $d=1$. In the present situation, assuming $\sum_{n \in \mathbb{Z}}\left|\varepsilon_{n}\right| / \rho_{n}<+\infty$, then for any value of $\sum_{s \in \mathbb{Z}} r_{s} \varepsilon_{s} /\left(p_{s} \rho_{s}\right)$, the random walk is recurrent.

Remark. Point iii) covers the situation considered in [12], where $\mathbb{P}\left(\mu_{n}=\delta_{1}\right)=\mathbb{P}\left(\mu_{n}=\right.$ $\left.\delta_{-1}\right)=1 / 2$ and $r_{n} /\left(p_{n}+q_{n}\right)=c$. When $\mathbb{P}\left(p_{0}=q_{0}\right)<1$, the rough critical scale for $\varepsilon_{n}$ with respect to recurrence/transience is $\exp \left(-|n|^{1 / 2}\right)$, so much smaller than for the vertically flat case (informally around $1 / n$ ). Remembering that the vertical random walk is Sinai's random walk, the intuition for this is easy. The landscape for $\left(S_{n}\right)$ somehow looks like a succession of horizontally invariant canyons, with transversal profile described by the potential of the vertical random walk. Then $\left(S_{n}\right)$ stays confined for a long time at the bottom of the canyons and just a little bit of horizontal flow is enough to make the random walk transient. The previous theorem quantifies this.

\section{Proof of the results}

\subsection{Preliminaries}

1) Relevant results from [2]. We group in a single theorem the results from [2], Theorem 2.4 and Proposition 2.51 ), used in the sequel. We rectify here a misleading point mentioned by the referee, appearing in [2] Definition 2.31 ), where the term "standard Lebesgue measure" on the half Euclidean ball $S_{+}^{d-1}=\left\{x \in \mathbb{R}^{d} \mid\|x\|=1, x_{1} \geq 0\right\}$ has to be replaced by "uniform probability measure". There is no problem when $d \geq 2$, but if $d=1$ this is $\delta_{1}$ instead of 0 (!). Notice that the form of the criterion comes from a polar decomposition ( $\operatorname{cf}[2]$, section 4.1, formula (8)).

Theorem 3.1. i) Let $d=2$. If $\exists \varepsilon>0$ so that for large $n$, $\Phi_{s t r}(n) \geq \sqrt{n}(\log n)^{1+\varepsilon}$, then $\left(S_{n}\right)$ is transient.

ii) Let $d=1$. A necessary and sufficient condition for recurrence is:

$$
\sum_{n \geq 1} \frac{1}{n^{2}} \frac{\left(\Phi^{-1}(n)\right)^{2}}{\Phi_{+}^{-1}(n)}=+\infty
$$

A sufficient condition for transience is $\sum_{n \geq 1} 1 / \Phi(n)<+\infty$.

2) Comparison of $\Phi_{s t r}, \Phi_{+}$and $\Phi$. One has $\Phi_{s t r} \preceq \Phi_{+} \preceq \Phi$ and more precisely:

$$
\Phi_{+}(n) \asymp \Phi_{s t r}(n)+\left(\sum_{-v_{-}^{-1}(n) \leq k \leq \ell \leq 0}^{\text {or } 0 \leq k \leq \ell \leq v_{+}^{-1}(n)} \rho_{k} \rho_{\ell}\left(\sum_{s=k}^{\ell} \frac{r_{s} \varepsilon_{s}}{p_{s} \rho_{s}}\right)^{2}\right)^{1 / 2},
$$


as well as:

$$
\Phi(n) \asymp \Phi_{s t r}(n)+\left(\sum_{-v_{-}^{-1}(n) \leq k \leq \ell \leq v_{+}^{-1}(n)} \rho_{k} \rho_{\ell}\left(\sum_{s=k}^{\ell} \frac{r_{s} \varepsilon_{s}}{p_{s} \rho_{s}}\right)^{2}\right)^{1 / 2} .
$$

Indeed, observe first that for $n \geq 0$ :

$$
\begin{aligned}
\sum_{0 \leq k \leq \ell \leq v_{+}^{-1}(n)} \rho_{k} \rho_{\ell}\left(1 / \rho_{k}^{2}+1 / \rho_{\ell}^{2}\right) & =\sum_{0 \leq k \leq \ell \leq v_{+}^{-1}(n)}\left(\rho_{k} / \rho_{\ell}+\rho_{\ell} / \rho_{k}\right) \\
& \asymp \sum_{0 \leq k \leq v_{+}^{-1}(n)} \rho_{k} \sum_{0 \leq \ell \leq v_{+}^{-1}(n)} 1 / \rho_{\ell} .
\end{aligned}
$$

Observe that $\sum_{0 \leq k \leq v_{+}^{-1}(n)} \rho_{k}=v_{+}\left(v_{+}^{-1}(n)\right) \leq n$ and:

$$
\begin{aligned}
n \leq v_{+}\left(v_{+}^{-1}(n)+1\right) & =v_{+}\left(v_{+}^{-1}(n)\right)+\rho_{v_{+}^{-1}(n)+1} \\
& \leq(1+1 / \eta) v_{+}\left(v_{+}^{-1}(n)\right) \leq(1+1 / \eta) n .
\end{aligned}
$$

Hence $v_{+}\left(v_{+}^{-1}(n)\right) \asymp n$ and we obtain:

$$
\sum_{0 \leq k \leq \ell \leq v_{+}^{-1}(n)} \rho_{k} \rho_{\ell}\left(1 / \rho_{k}^{2}+1 / \rho_{\ell}^{2}\right) \asymp n \sum_{0 \leq k \leq v_{+}^{-1}(n)} 1 / \rho_{k} .
$$

Proceeding in the same way for $\sum_{-v_{-}^{-1}(n) \leq k \leq \ell \leq 0}$ and summing, we get:

$$
\sum_{-v_{-}^{-1}(n) \leq k \leq \ell \leq 0 \text { or } 0 \leq k \leq \ell \leq v_{+}^{-1}(n)} \rho_{k} \rho_{\ell}\left(1 / \rho_{k}^{2}+1 / \rho_{\ell}^{2}\right) \asymp n \sum_{-v_{-}^{-1}(n) \leq k \leq v_{+}^{-1}(n)} 1 / \rho_{k}=\Phi_{s t r}^{2}(n) .
$$

This furnishes (3.2). In a very similar fashion:

$$
\begin{aligned}
\sum_{-v_{-}^{-1}(n) \leq k \leq \ell \leq v_{+}^{-1}(n)} \rho_{k} \rho_{\ell}\left(1 / \rho_{k}^{2}+1 / \rho_{\ell}^{2}\right) & \asymp \sum_{-v_{-}^{-1}(n) \leq k \leq v_{+}^{-1}(n)} \rho_{k} \sum_{-v_{-}^{-1}(n) \leq \ell \leq v_{+}^{-1}(n)} 1 / \rho_{\ell} \\
& \asymp n \sum_{-v_{-}^{-1}(n) \leq \ell \leq v_{+}^{-1}(n)} 1 / \rho_{\ell} \asymp \Phi_{\text {str }}^{2}(n) .
\end{aligned}
$$

This leads to (3.3).

3) The functions $\Phi_{s t r}^{-1}, \Phi_{+}^{-1}$ and $\Phi^{-1}$ check dominated variation. This property, coming from the structure of the main result (Theorem 2.4) in [2], considerably simplifies the study of the convergence of series. A non-decreasing function $f: \mathbb{R}_{+} \rightarrow \mathbb{R}_{+}$satisfies dominated variation if there exists a constant $C>0$ so that for large $x>0$ :

$$
f(2 x) \leq C f(x) .
$$

This implies that for any $A>0$, for $x>0$ large enough, $f(A x) \asymp f(x)$.

Dominated variation is obvious for $\Phi_{\text {str }}^{-1}$, because $\Phi_{s t r}(n)=\sqrt{n} \psi(n)$, with some nondecreasing $\psi$. This is a little less clear for $\Phi_{+}^{-1}$ and $\Phi^{-1}$ and shown in [2]. In fact in [2], using the same references and notations, some auxiliary functions $G_{u}$ and $G_{u,+}$ are defined in Definition 6.1 (where the involved $T_{k}^{l}(u)$ appear in Definition 5.1 and $\eta_{k}^{u}=r_{k}\left(\varepsilon_{k} . u\right) / p_{k}$ in Definitions 4.5 and 5.1) for which dominated variation of the inverses is shown in Lemma 6.2. As indicated at the end of section 6.5, $G_{u} \asymp \Phi_{u}$ and $G_{u,+} \asymp \Phi_{u,+}$ (see Definitions 2.3 and 6.1), so $\Phi_{u}^{-1}$ and $\Phi_{u,+}^{-1}$ check dominated variation as well. In our present situation, we just need to take $d=1$ and $u=1$ in order to have $\Phi_{u}=\Phi$ and $\Phi_{u,+}=\Phi_{+}$.

Notice on the contrary that, although well-defined, the function $v_{+}^{-1}(n)$ does not always check dominated variation in the non vertically flat case. 


\subsection{Proof of Proposition 2.1.}

As explained after the statement of the proposition, it remains to check transience when $d=2$ and $\mathbb{E}\left(\log \left(p_{0} / q_{0}\right)\right)=0$. When $\mathbb{P}\left(p_{0}=q_{0}\right)=1$, we have $\Phi_{s t r}(n) \succeq n$, so the condition for transience of Theorem $3.1 i)$ is verified. Consider next the case when $\mathbb{P}\left(p_{0}=q_{0}\right)<1$. We have $\operatorname{Var}\left(\log \left(p_{0} / q_{0}\right)\right)>0$. We shall repeatedly use that if $\left(Y_{n}\right)_{n \geq 1}$ is an i.i.d. sequence of non-constant random variables with $\mathbb{E}\left(Y_{1}^{2}\right)<+\infty$ and $\mathbb{E}\left(Y_{1}\right)=0$, then, a.-s., for any $\varepsilon>0$, for large $n$ :

$$
n^{1 / 2-\varepsilon} \leq \max _{1 \leq k \leq n}\left(Y_{1}+\cdots+Y_{k}\right) \leq n^{1 / 2+\varepsilon} .
$$

This may be seen for example as a consequence of classical results relative to the law of the Iterated Logarithm; see Chung [6], chap. 7 for instance.

Consider $\log \rho_{n}=\sum_{k=1}^{n} \log \left(q_{k} / p_{k}\right), n \geq 1$. We obtain that a.-s., for any $\varepsilon>0$, for $n$ large enough, $v_{+}(n) \leq n \exp \left(n^{1 / 2+\varepsilon}\right) \leq \exp \left(n^{1 / 2+2 \varepsilon}\right)$. Hence, using $1 /(1 / 2+2 \varepsilon) \geq$ $2(1-4 \varepsilon)$

$$
v_{+}^{-1}(n) \geq(\log n)^{2-9 \varepsilon} .
$$

As a result, using (3.5) for minima (i.e. with $-\log \left(q_{k} / p_{k}\right)$ ):

$$
\sum_{0 \leq k \leq v_{+}^{-1}(n)} 1 / \rho_{k} \geq \exp \left(\log ^{(2-9 \varepsilon)(1 / 2-\varepsilon)} n\right) \geq \exp \left(\log ^{1-7 \varepsilon} n\right) .
$$

Hence, a.-s., $\forall \varepsilon>0$, for $n$ large enough, $\Phi_{s t r}(n) \succeq \sqrt{n} \exp \left(\log ^{1-\varepsilon} n\right)$. Therefore the condition of Theorem $3.1 \mathrm{i}$ ) is also verified and $\left(S_{n}\right)$ is transient. This ends the proof of the proposition.

Remark. For the rest of the article, $d=1$ and $\mathbb{P}\left(p_{0}=q_{0}\right)<1$. For all the proofs of Theorem 2.5, we fix an integer $K>2(1+1 / \eta)^{2}$.

\subsection{Proof of Theorem $2.5 i$ )}

Consider point $i$ ) and $\left(\varepsilon_{n}\right)_{n \in \mathbb{Z}}$ verifying (2.1). Observe first that this condition holds if $\varepsilon_{n}=O\left(\exp \left(-|n|^{1 / 2+\delta}\right)\right), \delta>0$. Indeed, using (3.5), $\varepsilon_{n} / \rho_{n}=O\left(\exp \left(-|n|^{1 / 2+\delta / 2}\right)\right)$ in this case.

Now, condition (2.1) implies that $\sum_{u=k}^{\ell}\left(r_{s} \varepsilon_{s}\right) /\left(p_{s} \rho_{s}\right)$ is bounded in $(k, \ell), k \leq \ell$, since $\eta \leq r_{s} / p_{s} \leq 1 / \eta$. Using that (obtained as for getting (3.3)):

$$
\sum_{-v_{-}^{-1}(n) \leq k \leq \ell \leq v_{+}^{-1}(n)} \rho_{k} \rho_{\ell} \asymp n^{2},
$$

it then follows from (3.3) that $\Phi^{2}(n) \preceq \Phi_{s t r}^{2}(n)+n^{2}$ and so $\Phi(n) \preceq \Phi_{\text {str }}(n)+n$. As a result, because $\Phi^{-1}, \Phi_{\text {str }}^{-1}$ and $n \longmapsto n$ check dominated variation, we have $\Phi^{-1}(n) \succeq$ $\min \left\{\Phi_{\text {str }}^{-1}(n), n\right\}$. We shall also use that $\Phi_{+}^{-1}(n) \preceq \Phi_{\text {str }}^{-1}(n)$, coming from the general relation $\Phi_{+}(n) \succeq \Phi_{s t r}(n)$, consequence of (3.2) and dominated variation for $\Phi_{+}^{-1}$ and $\Phi_{\text {str }}^{-1}$.

In view of checking condition (3.1) in Theorem 3.1, given the previous remarks, it is sufficient to show the divergence of:

$$
\sum_{n \geq 1} \frac{1}{n^{2}} \frac{\left(\min \left\{\Phi_{\text {str }}^{-1}(n), n\right\}\right)^{2}}{\Phi_{\text {str }}^{-1}(n)}=\sum_{n \geq 1} \frac{1}{n} \min \left\{\frac{\Phi_{\text {str }}^{-1}(n)}{n}, \frac{n}{\Phi_{\text {str }}^{-1}(n)}\right\} .
$$

Splitting the sum according to the intervals $\left[K^{n}, K^{n+1}\right), n \geq 1$, and using that $\Phi_{\text {str }}^{-1}$ verifies dominated variation, the previous condition is equivalent to checking that:

$$
\sum_{n \geq 1} \min \left\{\frac{\Phi_{s t r}^{-1}\left(K^{n}\right)}{K^{n}}, \frac{K^{n}}{\Phi_{s t r}^{-1}\left(K^{n}\right)}\right\}=+\infty .
$$


Random walk in a stratified independent random environment

We prove below that the general term in the above series does not go to 0 .

Lemma 3.2. Almost-surely, $\limsup _{n \rightarrow+\infty} \frac{w_{+}(n)}{v_{+}(n)}=+\infty$ and $\limsup _{n \rightarrow+\infty} \min \left\{\frac{v_{+}(n)}{w_{+}(n)}, \frac{v_{-}(n)}{v_{+}(n)}, \frac{w_{+}(n)}{w_{-}(n)}\right\}=$ $+\infty$.

Proof of the lemma: Let us consider the second point. Fix an increasing sequence $\left(k_{n}\right)$ with $k_{n} / k_{n-1}^{2} \rightarrow+\infty$. For $n \in \mathbb{Z}$, let $R_{n}=\log \rho_{n}$. This is the potential of Sinaï's random walk. Let:

$$
U_{n}=\max _{k \in\left[-k_{n},-k_{n-1}\right)}\left(R_{k}-R_{-k_{n-1}}\right), V_{n}=\max _{k \in\left(k_{n-1}, k_{n}\right]}\left(R_{k}-R_{k_{n-1}}\right) .
$$

In the same way, introduce:

$$
W_{n}=\min _{k \in\left(k_{n-1}, k_{n}\right]}\left(R_{k}-R_{k_{n-1}}\right), X_{n}=\min _{k \in\left[-k_{n},-k_{n-1}\right)}\left(R_{k}-R_{-k_{n-1}}\right) .
$$

Let $\sigma>0$ be such that $\sigma^{2}=\operatorname{Var}\left(\log \left(q_{0} / p_{0}\right)\right)$. Let $c=2+2|\log (1 / \eta)|$ and notice that $\left|R_{k}\right| \leq$ $c|k|, k \in \mathbb{Z}$. Using functional convergence to standard Brownian motion $\left(B_{t}\right)_{t \in[-1,1]}$ :

$$
\begin{aligned}
& \mathbb{P}\left(\frac{U_{n}}{\sigma \sqrt{k_{n}-k_{n-1}}} \geq 1+\frac{V_{n}}{\sigma \sqrt{k_{n}-k_{n-1}}} \geq 2-\frac{W_{n}}{\sigma \sqrt{k_{n}-k_{n-1}}} \geq 3-\frac{X_{n}}{\sigma \sqrt{k_{n}-k_{n-1}}}\right) \\
& \longrightarrow{ }_{n \rightarrow+\infty} \mathbb{P}\left(\max _{t \in[-1,0]} B_{t} \geq 1+\max _{t \in[0,1]} B_{t} \geq 2-\min _{t \in[0,1]} B_{t} \geq 3-\min _{t \in[-1,0]} B_{t}\right)=: \alpha>0 .
\end{aligned}
$$

Using independence and the second Borel-Cantelli lemma, almost-surely the event appearing in the first probability is realized for infinitely many $n$. For such a $n$, we have:

$$
\begin{aligned}
v_{+}\left(k_{n}\right) & \geq \exp \left(-c k_{n-1}+\max _{k \in\left(k_{n-1}, k_{n}\right]}\left(R_{k}-R_{k_{n-1}}\right)\right) \\
& \geq \exp \left(-c k_{n-1}+\sigma \sqrt{k_{n}-k_{n-1}}-\min _{k \in\left(k_{n-1}, k_{n}\right]}\left(R_{k}-R_{k_{n-1}}\right)\right) \\
& \geq \exp \left(-3 c k_{n-1}+\sigma \sqrt{k_{n}-k_{n-1}}-\min _{k \in\left[1, k_{n}\right]} R_{k}\right) \\
& \geq \frac{w_{+}\left(k_{n}\right)}{k_{n}} \exp \left(-3 c k_{n-1}+\sigma \sqrt{k_{n}-k_{n-1}}\right) .
\end{aligned}
$$

If $n$ is large, $v_{+}\left(k_{n}\right) / w_{+}\left(k_{n}\right) \geq \exp \left(\sigma \sqrt{k_{n}} / 2\right)$, going to $+\infty$. Similar lower bounds are proved, with the same $n$, for $v_{-}\left(k_{n}\right) / v_{+}\left(k_{n}\right)$ and $w_{+}\left(k_{n}\right) / w_{-}\left(k_{n}\right)$. The first property stated in the lemma, namely $\lim \sup _{n \rightarrow+\infty} w_{+}(n) / v_{+}(n)=+\infty$, is proved in a similar (simpler) way.

We complete the proof. Using the first point of the previous lemma, a.-s., for any $A>0$, infinitely often, $w_{+}(n) \geq A v_{+}(n)$, i.e. $w_{+}\left(v_{+}^{-1}\left(v_{+}(n)\right)\right) \geq A v_{+}(n)$. Taking $\ell \in \mathbb{N}$ so that $\ell \leq v_{+}(n)<\ell+1$, we obtain:

$$
\Phi_{s t r}(\ell+1) \geq \sqrt{\ell+1} \sqrt{A \ell} \geq \ell \sqrt{A} .
$$

Hence $\Phi_{\text {str }}^{-1}(\ell \sqrt{A}) \leq \ell+1$. This implies that, a.-s., $\liminf \Phi_{\text {str }}^{-1}(n) / n=0$.

Using the second point of the previous lemma, a.-s., for any $A>1$, for infinitely many $n \in \mathbb{N}$, we have $v_{-}(n) \geq v_{+}(n) \geq A w_{+}(n)$ and $w_{+}(n) \geq w_{-}(n)$. For such a $n$, $v_{-}^{-1}\left(v_{+}(n)\right) \leq n$ and:

$$
\begin{aligned}
\frac{v_{+}(n)}{\sum_{-v_{-}^{-1}\left(v_{+}(n)\right) \leq k \leq v_{+}^{-1}\left(v_{+}(n)\right)} 1 / \rho_{k}} & \geq \frac{v_{+}(n)}{\sum_{-n \leq k \leq n} 1 / \rho_{k}} \geq \frac{v_{+}(n)}{a_{0} w_{-}(n)+w_{+}(n)} \\
& \geq \frac{v_{+}(n)}{w_{+}(n)}\left(1+a_{0}\right)^{-1} \geq \frac{A}{1+a_{0}} .
\end{aligned}
$$


Choosing $\ell \in \mathbb{N}$ such that $\ell \leq v_{+}(n)<\ell+1$, we obtain:

$$
\frac{\ell+1}{\Phi_{s t r}^{2}(\ell) / \ell} \geq \frac{A}{1+a_{0}}, \text { or } \Phi_{s t r}(\ell) \leq \frac{\sqrt{1+a_{0}}}{\sqrt{A}}(\ell+1)
$$

Hence $\Phi_{\text {str }}^{-1}\left(\sqrt{1+a_{0}}(\ell+1) / \sqrt{A}\right) \geq \ell$. Hence, a.-s., limsup $\Phi_{\text {str }}^{-1}(n) / n=+\infty$.

Let finally $b_{n}=\Phi_{\text {str }}^{-1}\left(K^{n}\right) / K^{n}$. Because $\Phi_{\text {str }}^{-1}$ checks dominated variation (3.4), a.-s., the previous results give $\liminf b_{n}=0$ and $\lim \sup b_{n}=+\infty$. Dominated variation also implies that, a.-s. for a constant $H>1$, for all $n$, we have $b_{n} / b_{n+1} \in[1 / H, H]$. Thus $b_{n} \in[1, H]$ for infinitely many $n$. For such a $n, \min \left\{b_{n}, 1 / b_{n}\right\}=1 / b_{n} \geq 1 / H$. This shows (3.8) and completes the proof of $i$ ).

\subsection{Proof of Theorem 2.5 $i i)$}

Assume here that the $\varepsilon_{n}$ are non-negative for large $n \in \mathbb{Z}$ and verify (2.2). The monotonicity of $C(n)$ for large $n$, hence of $n C(n)$, implies that the condition $\sum_{n \geq N_{0}} 1 /(n C(n))<$ $+\infty$ is equivalent to $\sum_{n \geq N_{1}} 1 / C\left(K^{n}\right)<+\infty$, for another $N_{1}>0$.

Let us first observe that $\sum_{n \geq N_{1}} 1 / C\left(K^{n}\right)<+\infty$, for some $N_{1}>0$, if there exists $\delta>0$ so that $\varepsilon_{n} \geq \exp \left(-|n|^{1 / 2-\delta}\right)$, for large $n \in \mathbb{Z}$. Indeed, using (3.5), a.-s., for any $\varepsilon>0$ and large $n, \exp \left(n^{1 / 2-\varepsilon}\right) \leq v_{+}(n) \leq \exp \left(n^{1 / 2+\varepsilon}\right)$. Therefore a.-s., for any $\varepsilon>0$ and large $n$, $(\log n)^{2+\varepsilon} \geq v_{+}^{-1}(n) \geq(\log n)^{2-\varepsilon}$. Via again (3.5), a.-s., for any $\varepsilon>0$, for $n$ large enough:

$$
\min _{0 \leq k \leq v_{+}^{-1}(n)} \rho_{k} \leq \exp \left(-\log ^{1-\varepsilon} n\right)
$$

Let $u_{n}$ be the first point in $\left[0, v_{+}^{-1}(n)\right]$ realizing the minimum of $\rho_{k}$ on this interval. Necessarily, $u_{n} \rightarrow+\infty$. Hence, a.-s., $\forall \varepsilon>0$, for large $n$, using that $u_{n} \leq v_{+}^{-1}(n) \leq$ $(\log n)^{2+\varepsilon}$, we obtain:

$$
\varepsilon_{u_{n}} / \rho_{u_{n}} \geq \exp \left(-u_{n}^{1 / 2-\delta}\right) \exp \left(\log ^{1-\varepsilon} n\right) \geq \exp \left(\log ^{1-2 \varepsilon} n\right)
$$

Hence, a.-s., $\forall \varepsilon>0$, for large $n, C(n) \geq(1 / 2) \varepsilon_{u_{n}} / \rho_{u_{n}} \geq \exp \left(\log ^{1-\varepsilon} n\right)$. Hence, a.-s., $\forall \varepsilon>0$, for large $n, C\left(K^{n}\right) \geq \exp \left(n^{1-\varepsilon}\right)$, giving $\sum_{n \geq N_{1}} 1 / C\left(K^{n}\right)<+\infty$.

We now prove transience for $\left(S_{n}\right)$, by checking the second condition in Theorem 3.1 ii), namely $\sum_{n \geq 1} 1 / \Phi(n)<+\infty$ or $\sum_{n \geq 1} K^{n} / \Phi\left(K^{n}\right)<+\infty$, as $\Phi$ is increasing.

Let $n_{0} \geq 0$ be such that $\varepsilon_{s} \geq 0$ whenever $s \geq v_{+}^{-1}\left(n_{0}\right)$ or $s \leq-v_{-}^{-1}\left(n_{0}\right)$. For large $n$ and $k \leq-v_{-}^{-1}\left(K^{n-1}\right)$ and $\ell \geq v_{+}^{-1}\left(K^{n-1}\right)$, using also that $\eta \leq r_{s} / p_{s} \leq 1 / \eta$, we have:

$$
\begin{aligned}
\sum_{k \leq s \leq \ell} \frac{r_{s} \varepsilon_{s}}{p_{s} \rho_{s}} & \geq \sum_{-v_{-}^{-1}\left(K^{n-1}\right) \leq s \leq v_{+}^{-1}\left(K^{n-1}\right)} \frac{r_{s} \varepsilon_{s}}{p_{s} \rho_{s}} \\
& \geq \sum_{s \in\left[-v_{-}^{-1}\left(K^{n-1}\right), v_{+}^{-1}\left(K^{n-1}\right)\right] \backslash\left[-v_{-}^{-1}\left(n_{0}\right), v_{+}^{-1}\left(n_{0}\right)\right]} \frac{r_{s} \varepsilon_{s}}{p_{s} \rho_{s}}+\sum_{s \in\left[-v_{-}^{-1}\left(n_{0}\right), v_{+}^{-1}\left(n_{0}\right)\right]} \frac{r_{s} \varepsilon_{s}}{p_{s} \rho_{s}} \\
& \geq \sum_{s \in\left[-v_{-}^{-1}\left(K^{n-1}\right), v_{+}^{-1}\left(K^{n-1}\right)\right] \backslash\left[-v_{-}^{-1}\left(n_{0}\right), v_{+}^{-1}\left(n_{0}\right)\right]} \frac{\varepsilon_{s}}{\rho_{s}}+\sum_{s \in\left[-v_{-}^{-1}\left(n_{0}\right), v_{+}^{-1}\left(n_{0}\right)\right]} \frac{r_{s}}{p_{s} \rho_{s}} \\
& \geq(\eta / 2) \sum_{s \in\left[-v_{-}^{-1}\left(K^{n-1}\right), v_{+}^{-1}\left(K^{n-1}\right)\right]} \sum_{\rho_{s}}=(\eta / 2) C\left(K^{n-1}\right)>0 .
\end{aligned}
$$


We obtain that for large $n \geq 0$ :

$$
\begin{aligned}
\Phi^{2}\left(K^{n}\right) & \sum_{\substack{-v_{-}^{-1}\left(K^{n}\right) \leq k<-v_{-}^{-1}\left(K^{n-1}\right) \\
v_{+}^{-1}\left(K^{n-1}\right)<\ell \leq v_{+}^{-1}\left(K^{n}\right)}} \rho_{k} \rho_{\ell}\left(\sum_{k \leq s \leq \ell} \frac{r_{s} \varepsilon_{s}}{p_{s} \rho_{s}}\right)^{2} \\
& \left(C\left(K^{n-1}\right)\right)^{2} \sum_{\substack{-v_{-}^{-1}\left(K^{n}\right) \leq k<-v_{-}^{-1}\left(K^{n-1}\right) \\
v_{+}^{-1}\left(K^{n-1}\right)<\ell \leq v_{+}^{-1}\left(K^{n}\right)}} \rho_{k} \rho_{\ell} .
\end{aligned}
$$

$\operatorname{Next} \sum_{k=v_{+}^{-1}\left(K^{n-1}\right)+1}^{v_{+}^{-1}\left(K^{n}\right)} \rho_{k}=v_{+}\left(v_{+}^{-1}\left(K^{n}\right)\right)-v_{+}\left(v_{+}^{-1}\left(K^{n-1}\right)\right) \leq v_{+}\left(v_{+}^{-1}\left(K^{n}\right)\right) \leq K^{n}$. Also:

$$
v_{+}\left(v_{+}^{-1}\left(K^{n}\right)\right)-v_{+}\left(v_{+}^{-1}\left(K^{n-1}\right)\right) \geq \frac{K^{n}}{1+1 / \eta}-K^{n-1} \geq \frac{K^{n}}{2(1+1 / \eta)},
$$

using that $K>2(1+1 / \eta)$. In the same way:

$$
\begin{aligned}
\sum_{-v_{-}^{-1}\left(K^{n}\right) \leq k<-v_{-}^{-1}\left(K^{n-1}\right)} \rho_{k} & =\frac{1}{a_{0}}\left[v_{-}\left(v_{-}^{-1}\left(K^{n}\right)-1\right)-v_{-}\left(v_{-}^{-1}\left(K^{n-1}\right)-1\right)\right] \\
& \leq \frac{1}{\eta} v_{-}\left(v_{-}^{-1}\left(K^{n}\right)\right) \leq \frac{K^{n}}{\eta} .
\end{aligned}
$$

On the other hand, because $v_{-}(p) \geq v_{-}(p+1) /(1+1 / \eta)$, the left-hand side is:

$$
\geq \eta\left(\frac{v_{-}\left(v_{-}^{-1}\left(K^{n}\right)+1\right)}{(1+1 / \eta)^{2}}-K^{n-1}\right) \geq \eta\left(\frac{K^{n}}{(1+1 / \eta)^{2}}-K^{n-1}\right) \geq \eta \frac{K^{n}}{2(1+1 / \eta)^{2}}
$$

Consequently, $\Phi\left(K^{n}\right) \succeq K^{n} C\left(K^{n-1}\right)$. The hypothesis $\sum_{n \geq N_{1}} 1 / C\left(K^{n}\right)<+\infty$ therefore implies that $\sum_{n \geq 1} K^{n} / \Phi\left(K^{n}\right)<+\infty$. This completes the proof.

\subsection{Proof of Theorem $2.5 \mathrm{iii}$ )}

Here the $\left(\varepsilon_{n}\right)_{\in \mathbb{Z}}$ are independent random variables, independent from the $\left(p_{n}, q_{n}, r_{n}\right)_{n \in \mathbb{Z}}$, under the conditions of point $\left.i i i\right)$ of the theorem.

First of all, as for getting (3.9) and (3.10) for instance:

$$
\sum_{v_{+}^{-1}\left(K^{n-1}\right)<\ell \leq v_{+}^{-1}\left(K^{n}\right)} \rho_{\ell} \asymp K^{n} \text { and } \sum_{-v_{-}^{-1}\left(K^{n}\right) \leq k \leq-1} \rho_{k} \asymp K^{n} .
$$

In order to control the proportion of $(k, \ell)$ in the definition of $\Phi\left(K^{n}\right)$ such that $\sum_{k \leq s \leq \ell}\left(r_{s} \varepsilon_{s} /\left(p_{s} \rho_{s}\right)\right)$ is large, we introduce the following probability measure on $\mathbb{Z}^{2}$ :

$$
\nu_{n}=\frac{1}{Z_{n}} \sum_{-v_{-}^{-1}\left(K^{n}\right) \leq k \leq-1, v_{+}^{-1}\left(K^{n-1}\right)<\ell \leq v_{+}^{-1}\left(K^{n}\right)} \rho_{k} \rho_{\ell} \delta_{(k, \ell)}
$$

where the normalizing constant $Z_{n}$ thus verifies $Z_{n} \asymp K^{2 n}$.

It follows from (3.5) that, a.-s., for all $\varepsilon>0$, for large $n,(\log n)^{2-\varepsilon} \leq v_{+}^{-1}(n) \leq$ $(\log n)^{2+\varepsilon}$. Hence, a.-s., for all $\varepsilon>0$, for large $n, n^{2-\varepsilon} \leq v_{+}^{-1}\left(K^{n}\right) \leq n^{2+\varepsilon}$. Let now $u_{n} \in\left[0, v_{+}^{-1}\left(K^{n-1}\right)\right]$ be the first minimum of $\rho_{k}$ on this interval. Then, from (3.5), a.-s., $\forall \varepsilon>0, \forall \varepsilon^{\prime}>0$ :

$$
\exp \left(u_{n}^{1 / 2+\varepsilon^{\prime}}\right) \geq \max _{0 \leq k \leq u_{n}} \frac{1}{\rho_{k}} \geq \frac{1}{\rho_{u_{n}}} \geq \exp \left(n^{1-\varepsilon}\right)
$$


for large $n$. Hence, a.-s., $\forall \varepsilon>0$, for large $n, n^{2+\varepsilon} \geq v_{+}^{-1}\left(K^{n-1}\right) \geq u_{n} \geq n^{2-\varepsilon}$.

Let $\delta / 2>\gamma>2 \delta / 5$. We next assume that $\varepsilon>0$ is small enough so that $\gamma+(2+$ $\varepsilon)(1 / 2-\delta) \leq 1-3 \varepsilon$ and $(2+\varepsilon) \delta / 5<\gamma<\delta / 2$.

Recall that, a.-s., $\varepsilon_{n} \in[-1 / \eta, 1 / \eta]$. Let $C_{0}=2 / \eta+1$ and $l(n)=n^{\gamma}$. Starting from $u_{n, 0}=u_{n}$ and going left, we shall successively choose in a decreasing order in the interval $\left[0, u_{n}\right]$, points $u_{n, 0}>u_{n, 1}>\cdots>u_{n, l(n)}>0$, with increasing $\rho_{u_{n, 0}}<\cdots<\rho_{u_{n, l(n)}}$. For $0 \leq \ell<l(n)$, define $u_{n, \ell+1}$ as the closest point on the left side of $u_{n, \ell}$ with:

$$
\left(\frac{1}{4} \frac{C_{0} / \eta^{2}}{\rho_{u_{n, \ell}}}>\right) \frac{1}{4} \frac{\exp \left(-u_{n, \ell}^{1 / 2-\delta}\right)}{\rho_{u_{n, \ell}}} \geq \frac{C_{0} / \eta^{2}}{\rho_{u_{n, \ell+1}}} \geq \frac{1}{4 K} \frac{\exp \left(-u_{n, \ell}^{1 / 2-\delta}\right)}{\rho_{u_{n, \ell}}}
$$

We have used for the last two inequalities that $\rho_{k} / \rho_{k+1} \in[1 / K, K]$, for $k \in \mathbb{Z}$. We recursively check that for $0 \leq \ell<l(n), u_{n, \ell+1}$ is a well-defined point in $\left(0, u_{n, \ell}\right)$, since:

$$
\begin{aligned}
\rho_{u_{n, \ell+1}} \leq\left(4 C_{0} K / \eta^{2}\right) \rho_{u_{n, \ell}} \exp \left(u_{n, \ell}^{1 / 2-\delta}\right) & \leq\left(4 C_{0} K / \eta^{2}\right)^{l(n)} \rho_{u_{n, 0}} \exp \left(l(n) u_{n, 0}^{1 / 2-\delta}\right) \\
& \leq \exp \left(n^{\gamma} \log \left(4 C_{0} K / \eta^{2}\right)-n^{1-\varepsilon}+n^{\gamma} n^{(2+\varepsilon)(1 / 2-\delta)}\right) \\
& \leq \exp \left(-n^{1-2 \varepsilon}\right)
\end{aligned}
$$

for large $n$. The last upper-bound is uniform in $0 \leq \ell<l(n)$. In particular, for large $n$ :

$$
\rho_{u_{n, l(n)}} \leq \exp \left(-n^{1-2 \varepsilon}\right)
$$

Since $u_{n, l(n)}>0$, this implies that $u_{n, l(n)} \rightarrow+\infty$. Remark also that conditions (3.11) imply that $\rho_{u_{n, \ell+1}} \geq 4 \rho_{u_{n, \ell}}$, for $0 \leq \ell<l(n)$.

We now reason conditionally to the $\left(p_{n}, q_{n}, r_{n}\right)_{n \in \mathbb{Z}}$ and make a measurable construc-

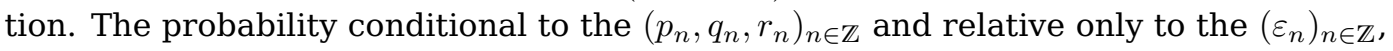
is written as $\mathbb{P}^{\prime}$, with corresponding expectation $\mathbb{E}^{\prime}$. Consider the event $A_{n}$, where $\left(\varepsilon_{i}\right)_{i \in \mathbb{Z}} \in A_{n}$ if:

$$
\nu_{n}\left\{(k, \ell) \in \mathbb{Z}^{2},\left|\sum_{k \leq u \leq \ell} \frac{r_{u} \varepsilon_{u}}{p_{u} \rho_{u}}\right| \leq \frac{C_{0}}{\eta \rho_{u_{n, l(n)}}}\right\} \geq 3 / 4
$$

Let us write:

$$
\mathbb{P}^{\prime}\left(A_{n}\right)=\mathbb{E}^{\prime}\left(\mathbb{E}^{\prime}\left(1_{\left(\varepsilon_{k}\right)_{k \in \mathbb{Z}} \in A_{n}} \mid\left(\varepsilon_{\ell}\right)_{\ell \neq u_{n, s}, 0 \leq s<l(n)}\right)\right) .
$$

Fixing $\left(\varepsilon_{\ell}\right)_{\ell \neq u_{n, s}, 0 \leq s<l(n)}$, suppose that there exists $\left(\varepsilon_{u_{n, s}}\right)_{0 \leq s<l(n)}$ with $\varepsilon=\left(\varepsilon_{i}\right)_{i \in \mathbb{Z}} \in A_{n}$. Take next any $\left(\varepsilon_{u_{n, s}}^{\prime}\right)_{0 \leq s<l(n)} \neq\left(\varepsilon_{u_{n, s}}\right)_{0 \leq s<l(n)}$ and call $\varepsilon^{\prime}$ the point obtained from $\varepsilon$ by replacing $\left(\varepsilon_{u_{n, s}}\right)_{0 \leq s<l(n)}$ by $\left(\varepsilon_{u_{n, s}}^{\prime}\right)_{0 \leq s<l(n)}$, without changing the other coordinates.

We claim that $\varepsilon^{\prime} \notin A_{n}$. Indeed, let $0 \leq p<l(n)$ be the smallest index so that $\varepsilon_{u_{n, p}}^{\prime} \neq \varepsilon_{u_{n, p}}$. Take any $(k, \ell)$ in the set involved in (3.13) such that $\nu_{n}(k, \ell)>0$ and notice that $k<0$ and $v_{+}^{-1}\left(K^{n-1}\right)<\ell$. Using the hypothesis on the laws of the $\varepsilon_{n}$, that $\eta \leq r_{u} / p_{u} \leq 1 / \eta, u \in \mathbb{Z}$, and the conditions in (3.11), we have:

$$
\begin{aligned}
\left|\sum_{k \leq u \leq \ell} \frac{r_{u} \varepsilon_{u}^{\prime}}{p_{u} \rho_{u}}\right| & =\left|\frac{r_{u_{n, p}}}{p_{u_{n, p}}} \frac{\varepsilon_{u_{n, p}}^{\prime}-\varepsilon_{u_{n, p}}}{\rho_{u_{n, p}}}+\sum_{p<q<l(n)} \frac{r_{u_{n, q}}}{p_{u_{n, q}}} \frac{\varepsilon_{u_{n, q}}^{\prime}-\varepsilon_{u_{n, q}}}{\rho_{u_{n, q}}}+\sum_{k \leq u \leq \ell} \frac{r_{u}}{p_{u}} \frac{\varepsilon_{u}}{\rho_{u}}\right| \\
& \geq \eta \frac{\exp \left(-u_{n, p}^{1 / 2-\delta}\right)}{\rho_{u_{n, p}}}-\sum_{p<q<l(n)} \frac{C_{0}}{\eta \rho_{u_{n, q}}}-\frac{C_{0}}{\eta \rho_{u_{n, l(n)}}} \\
& \geq 4 \frac{C_{0}}{\eta \rho_{u_{n, p+1}}}-\frac{C_{0}}{\eta \rho_{u_{n, p+1}}} \sum_{r \geq 0} 4^{-r} \geq 2 \frac{C_{0}}{\eta \rho_{u_{n, p+1}}} \geq 2 \frac{C_{0}}{\eta \rho_{u_{n, l(n)}}}
\end{aligned}
$$


This being true for any $(k, \ell)$ appearing in the set in (3.13) with $\nu_{n}(k, \ell)>0$, this furnishes:

$$
\nu_{n}\left\{(k, \ell) \in \mathbb{Z}^{2},\left|\sum_{k \leq u \leq \ell} \frac{r_{u} \varepsilon_{u}^{\prime}}{p_{u} \rho_{u}}\right| \leq \frac{C_{0}}{\eta \rho_{u_{n, l(n)}}}\right\} \leq 1 / 4 .
$$

Hence $\varepsilon^{\prime} \notin A_{n}$. As a result, for large $n$, using (2.3), independence, $u_{n} \leq n^{2+\varepsilon}$ and at the end $1-x \leq e^{-x}, x \in \mathbb{R}$ :

$$
\begin{aligned}
\mathbb{E}^{\prime}\left(1_{\left(\varepsilon_{k}\right)_{k \in \mathbb{Z}} \in A_{n}} \mid\left(\varepsilon_{\ell}\right)_{\ell \neq u_{n, s}, 0 \leq s<l(n)}\right) & \leq\left(1-u_{n, 0}^{-\delta / 5}\right) \cdots\left(1-u_{n, l(n)-1}^{-\delta / 5}\right) \\
& \leq\left(1-u_{n}^{-\delta / 5}\right)^{l(n)} \leq \exp \left(-n^{\gamma-(2+\varepsilon) \delta / 5}\right) .
\end{aligned}
$$

By (3.14), we deduce that $\mathbb{P}^{\prime}\left(A_{n}\right) \leq \exp \left(-n^{\gamma-(2+\varepsilon) \delta / 5}\right)$. Now $\gamma-(2+\varepsilon) \delta / 5>0$, leading to $\sum \mathbb{P}^{\prime}\left(A_{n}\right)<\infty$. By (3.13) and the first Borel-Cantelli lemma, for $\mathbb{P}^{\prime}$-almost-all $\left(\varepsilon_{i}\right)_{i \in \mathbb{Z}}$, we obtain for large $n$ :

$$
\nu_{n}\left\{(k, \ell) \in \mathbb{Z}^{2},\left|\sum_{k \leq u \leq \ell} \frac{r_{u} \varepsilon_{u}}{p_{u} \rho_{u}}\right| \geq \frac{C_{0}}{\eta \rho_{u_{n, l(n)}}}\right\} \geq 1 / 4 .
$$

We now conclude the argument. First, write $\mathcal{E}_{n}(f(X, Y))$ for $\sum_{(k, \ell) \in \mathbb{Z}^{2}} f(k, \ell) \nu_{n}(k, \ell)$. We shall check the second condition of Theorem $3.1 \mathrm{ii}$ ) for proving transience of $\left(S_{n}\right)$, i.e. $\sum_{n>1} 1 / \Phi(n)<+\infty$, or equivalently $\sum_{n>1} K^{n} / \Phi\left(K^{n}\right)<+\infty$, as $\Phi(n)$ is increasing.

As a result, from (3.17), $\mathbb{P}^{\prime}$-a.-s., there exists $N_{0} \geq 0$, so that for $n \geq N_{0}$ :

$$
\mathcal{E}_{n}\left[\left(\sum_{X \leq u \leq Y} \frac{r_{u} \varepsilon_{u}}{p_{u} \rho_{u}}\right)^{2}\right] \geq \frac{1}{4} \frac{C_{0}^{2}}{\eta^{2} \rho_{u_{n, l(n)}}^{2}} .
$$

This then furnishes:

$$
\begin{aligned}
\sum_{n \geq N_{0}} \frac{K^{n}}{\Phi\left(K^{n}\right)} & \leq \sum_{n \geq N_{0}} K^{n}\left(\sum_{-v_{-}^{-1}\left(K^{n}\right) \leq k \leq \ell \leq v_{+}^{-1}\left(K^{n}\right)} \rho_{k} \rho_{\ell}\left(\sum_{k \leq u \leq \ell} \frac{r_{u} \varepsilon_{u}}{p_{u} \rho_{u}}\right)^{2}\right)^{-1 / 2} \\
& \leq \sum_{n \geq N_{0}} K^{n}\left(\sum_{-v_{-}^{-1}\left(K^{n}\right) \leq k \leq-1, v_{+}^{-1}\left(K^{n-1}\right)<\ell \leq v_{+}^{-1}\left(K^{n}\right)} \rho_{k} \rho_{\ell}\left(\sum_{k \leq u \leq \ell} \frac{r_{u} \varepsilon_{u}}{p_{u} \rho_{u}}\right)^{2}\right)^{-1 / 2} \\
& \leq \sum_{n \geq N_{0}} \frac{K^{n}}{\left(Z_{n}\right)^{1 / 2}}\left(\mathcal{E}_{n}\left[\left(\sum_{X \leq u \leq Y} \frac{r_{u} \varepsilon_{u}}{p_{u} \rho_{u}}\right)^{2}\right]\right)^{-1 / 2} \\
& \leq \sum_{n \geq N_{0}} \frac{K^{n}}{\left(Z_{n}\right)^{1 / 2}} \frac{2 \eta \rho_{u_{n, l(n)}} \preceq \sum_{n \geq N_{0}} \rho_{u_{n, l(n)}}<+\infty}{C_{0}}
\end{aligned}
$$

as from (3.12), we have $\rho_{u_{n, l(n)}} \leq \exp \left(-n^{1-2 \varepsilon}\right)$. Hence $\sum_{n \geq 1} K^{n} / \Phi\left(K^{n}\right)<+\infty$ and the proof of point iii) of the theorem is complete.

Remark. Let us finally discuss the expected normalization for $\left(S_{n}\right)$, taking $d=1$ and the context of Theorem 2.5. Introduce as in [1, 2] the random times $0=\sigma_{0}<\tau_{0}<\sigma_{1}<$ $\tau_{1}<\cdots$, where $\tau_{k}=\min \left\{n>\sigma_{k} \mid S_{n} \notin \mathbb{Z} \times\{0\}\right\}$ and $\sigma_{k+1}=\min \left\{n>\tau_{k} \mid S_{n} \in \mathbb{Z} \times\{0\}\right\}$. Setting $D_{n}=S_{\sigma_{n}}-S_{\sigma_{n-1}}$, conditionally on the environment, the $\left(D_{n}\right)_{n \geq 1}$ are $i . i . d$.

Standardly (cf for example Gnedenko-Kolmogorov [8]), the correct normalization for $D_{1}+\cdots+D_{n}$ can be read on the behaviour at the origin of the characteristic function 
$\chi_{D_{1}}(t)=\mathbb{E}\left(e^{i t D_{1}}\right), t \in \mathbb{R}$. More precisely, if $m_{N}$ is the empirical mean and $d_{N}^{2}$ the empirical variance of $\left(D_{1}+\cdots+D_{n}\right)_{1 \leq n \leq N}$, then $d_{N}$ and $\left|m_{N}\right|+d_{N}$ are respectively related to $1-\operatorname{Re}\left(\chi_{D}(t)\right)$ and $\left|1-\chi_{D}(t)\right|$, as $t \rightarrow 0$. By [2], Prop. 6.5 and Prop. 6.8, one may informally guess that $d_{N}$ is like $\Phi_{+}(N)$ and $\left|m_{N}\right|+d_{N}$ like $\Phi(N)$. In the special case when $\varepsilon_{n}=1$, the correct normalization for $\sigma_{n}$ should be $\tilde{\Phi}(n)$, with:

$$
\tilde{\Phi}(n) \asymp\left(\sum_{-v_{-}^{-1}(n) \leq k \leq \ell \leq v_{+}^{-1}(n)} \rho_{k} \rho_{\ell}\left(\sum_{s=k}^{\ell} \frac{1}{\rho_{s}}\right)^{2}\right)^{1 / 2} .
$$

One may notice that $\tilde{\Phi}^{-1}(n) \asymp \Psi^{-1}(n)$, where $\Psi(n)=n \sum_{-v_{-}^{-1}(n) \leq k \leq v_{+}^{-1}(n)} 1 / \rho_{k}$. A natural conjecture for the normalization of $S_{n}$ is then:

$$
\left(\Phi \circ \Psi^{-1}(n), \Psi^{-1}(n)\right) .
$$

Since $\Psi^{-1}(n)$ has rough order $(\log n)^{2}$, one finds in the second coordinate the scaling of Sinaï's random walk [17]. Recall that in the vertically flat case and when the $\left(r_{n} \varepsilon_{n} / p_{n}\right)_{n \in \mathbb{Z}}$ are i.i.d. centered and non-constant, then $\Phi(n) \asymp n^{3 / 2}$ and $\Psi(n) \asymp n^{2}$. From these informal considerations, one recovers that the correct normalization for $S_{n}$ in this case is $\left(n^{3 / 4}, \sqrt{n}\right)$; see [7] and [10] for more precise informations.

Acknowledgments. We thank Jacques Printems and Alexis Devulder for useful discussions, as well as Françoise Pène for mentioning reference [12]. We are also grateful to the anonymous referee for numerous remarks leading to many improvements of the first versions of this article.

\section{References}

[1] J. Brémont, On planar random walks in environments invariant by horizontal translations, Markov Processes and Related Fields 2016, issue 2, vol. 22, 267-310. MR-3561139

[2] J. Brémont, Markov chains in a stratified environment, ALEA, Lat. Am. J. Probab. Math. Stat. 14, 751-798 (2017). MR-3704410

[3] M. Campanino, D. Petritis, Random walks on randomly oriented lattices, Markov Processes and Related Fields 9 (2003), no. 3, 391-412. MR-2028220

[4] M. Campanino, D. Petritis, Type transitions of simple random walks on randomly directed regular lattices, Journal of Applied Probability 51, 1065-1080 (2014). MR-3301289

[5] F. Castell, N. Guillotin, F. Pene, B. Schapira, A local limit theorem for random walks in random scenery and on randomly oriented lattices, Annals of Proba. 39 (2011), no. 6, 2079-2118. MR-2932665

[6] K. Chung, A course in probability theory. Second edition. Academic Press.1974. MR-0346858

[7] A. Devulder, F. Pene, Random walk in random environment in a two-dimensional stratified medium with orientations, Electron. Journal of Probab. 18 (2013), no. 88, 1-23. MR-3035746

[8] B. V. Gnedenko, A. N. Kolmogorov, Limit distributions for sums of independent random variables, Addison-Wesley Publishing Company, revised edition, 1968. MR-0233400

[9] N. Guillotin-Plantard, A. Le Ny, Transient random walks on $2 D$-oriented lattices, Theory Probab. Appl. 52 (2008), no. 4, 699-711. MR-2742878

[10] N. Guillotin-Plantard, A. Le Ny, A functional limit theorem for a $2 d$ - random walk with dependent marginals. Electronic Communications in Probability (2008), Vol. 13, 337-351. MR-2415142

[11] S. Kalikow, Generalized random walk in a random environment. Ann. Probab. 9, 753-768. MR-0628871 
Random walk in a stratified independent random environment

[12] M. Kochler, Random walks in random environment: random orientations and branching, Ph-D thesis, Technische Universität München, 2012.

[13] M. Matheron, G. de Marsily, Is transport in porous media always diffusive? A counterexample. Water resources Res. 16:901-907, 1980.

[14] D. Ornstein, Random walks. I, II. Trans. Amer. Math. Soc. 138 (1969), 1-43; ibid. 138 (1969), 45-60. MR-0238399

[15] F. Pene, Transient random walk in $\mathbb{Z}^{2}$ with stationary orientations, ESAIM Probability and Statistics 13 (2009), 417-436. MR-2554964

[16] C. Sabot, Random Walks in Random Dirichlet Environment are transient in dimension $d \geq 3$, Probab. Theory Related Fields 151 (2011), no. 1-2, 297-317. MR-2834720

[17] Y. Sinaï, The limit behavior of a one-dimensional random walk in a random environment, Teor. Veroyatnost. i Primenen. 27 (1982), no. 2, 247-258. MR-0657919

[18] F. Solomon, Random walks in a random environment. Ann. Probability 3 (1975), 1-31. MR0362503

[19] F. Spitzer, Principles of random walks. Second edition. Graduate Texts in Mathematics, vol. 34. Springer-Verlag, New-York Heidelberg, 1976. MR-0388547 


\section{Electronic Journal of Probability Electronic Communications in Probability}

\section{Advantages of publishing in EJP-ECP}

- Very high standards

- Free for authors, free for readers

- Quick publication (no backlog)

- Secure publication $\left(\mathrm{LOCKSS}^{1}\right)$

- Easy interface (EJMS²)

\section{Economical model of EJP-ECP}

- Non profit, sponsored by $\mathrm{IMS}^{3}, \mathrm{BS}^{4}$, ProjectEuclid ${ }^{5}$

- Purely electronic

\section{Help keep the journal free and vigorous}

- Donate to the IMS open access fund ${ }^{6}$ (click here to donate!)

- Submit your best articles to EJP-ECP

- Choose EJP-ECP over for-profit journals

\footnotetext{
${ }^{1}$ LOCKSS: Lots of Copies Keep Stuff Safe http://www. lockss.org/

${ }^{2}$ EJMS: Electronic Journal Management System http://www.vtex.lt/en/ejms.html

${ }^{3}$ IMS: Institute of Mathematical Statistics http://www.imstat.org/

${ }^{4}$ BS: Bernoulli Society http://www. bernoulli-society.org/

${ }^{5}$ Project Euclid: https://projecteuclid.org/

${ }^{6}$ IMS Open Access Fund: http://www.imstat.org/publications/open.htm
} 